\title{
Die Arbeitsgemeinschaft der Regionalbibliotheken in der Sektion IV des DBV
}

\author{
Axel Halle
}

Die AG hat zwei Wurzeln: einerseits die 1958 gegründete Arbeitsgemeinschaft der Landesbibliotheken und andererseits die 1963 gebildete Arbeitsgemeinschaft Kommunaler Wissenschaftlicher Bibliotheken. Beide Arbeitsgemeinschaften fusionierten 1971 zur AG der Regionalbibliotheken. Die fachliche Arbeit erfolgte bis Mitte der siebziger Jahre unter dem Dach des VDB. Mit der Übernahme der bibliothekarischen Sacharbeit durch den DBV erfolgte 1985 die Integration der AG der Regionalbibliotheken in die Sektion IV.

Von den knapp 70 einschlägigen bundesdeutschen Bibliotheken sind 60 Mitglieder der AG. Nicht alle Mitglieder sind in der Sektion IV, einige gehören anderen Sektionen an. Zusätzlich gehören zwei Österreichische und eine Schweizer Regionalbibliothek der AG an. Die AG ist lose organisiert, besitzt weder Satzung noch Geschäftsordnung und erhebt keine Mitgliedsbeiträge.

Die Gruppe ist heterogen: Es gibt Landesbibliotheken, Staatsbibliotheken oder Staatliche Bibliotheken unterschiedlichster Größe, regionaler Zuständigkeiten, mit und ohne Pflichtexemplarrecht etc. Beispielsweise stehen neben den großen Landesbibliotheken in Stuttgart, Karlsruhe oder Hannover kleine in Eutin oder Dessau. Daneben gibt es wissenschaftliche Stadtbibliotheken, z. B. Trier oder Lübeck, Mischformen von Staats- und Stadtbibliotheken, beispielsweise Augsburg und Potsdam, aber auch von wissenschaftlichen und öffentlichen Stadtbibliotheken, z. B. ZLB Berlin. Neben den „reinen“ Regionalbibliotheken gibt es eine Reihe von Bibliotheken, die neben ihrem regionalen Bezug vor allem institutionelle Informationsversorgungsfunktionen haben. Hier ist an die größere Zahl von Universitätsund Landesbibliotheken zu denken, bei denen der Regionalbezug einen unterschiedlich großen Anteil hat. Allerdings gibt es auch "reine" Regionalbibliotheken, die für die örtliche Universität oder Fachhochschule, mindestens für bestimmte Fachgebiete, Kernaufgaben der Literaturversorgung übernehmen. Last but not least sind einige Forschungsbibliotheken Mitglieder der AG, die in ihrem historischen Kern landesherrliche Wurzeln und damit einen engen Regionalbezug haben, z. B. Herzogin Anna Amalia Bibliothek oder Herzog August Bibliothek.

Eine wichtige Gruppe innerhalb der AG ist die seit 1982 existierende Arbeitsgruppe Regionalbibliographie. An ihr sind alle Bibliotheken personell beteiligt, die Regionalbibliographien erarbeiten. Seit Jahren wird diese Arbeit sehr erfolgreich durch Herrn Syré koordiniert und geleitet. 
Die Stellung der Regionalbibliotheken wird unter anderem im Wissenschaftsratsgutachten 1964, im Bibliotheksplan 73 und in Bibliotheken ' 93 dokumentiert. Die Arbeit der Regionalbibliotheken fand in den 1971 und 2000 erschienen Sonderheften der Zeitschrift für Bibliothekswesen und Bibliographie Niederschlag. Darüber hinaus hat die AG ein Sonderheft über Bibliotheksbau von Regionalbibliotheken verantwortet. In Kürze wird ein Sonderheft über die Regionalbibliographien im deutschen Sprachraum, ebenfalls als Sonderheft der ZfBB, erscheinen.

Der fünfköpfige Vorstand setzt sich derzeit wie folgt zusammen: 2. Vorsitzender Dr. Ehrle, 3. Vorsitzende Frau Dr. Dörr, 1. Vorsitzender Dr. Halle. Jedes dieser Ämter wird - analog der Regelungen des VDB - seit einigen Jahren üblicherweise für zwei Jahre wahrgenommen. Um Kontinuität zu gewährleisten, erfolgt die Wahl zunächst für das Amt des dritten Vorsitzenden, der oder die dann nach zwei Jahren den Vorsitz übernimmt und dann anschließend weitere zwei Jahre dem Vorstand angehört. Die Regionalbibliographien sind durch deren Sprecher, Dr. Syré, im Vorstand vertreten. Schriftführer ist Herr Hellfaier.

Die AG führt jährlich zwei Treffen durch: ein eineinhalbtägiges im Herbst, dieses Jahr am 24. und 25. Oktober in Kassel, und ein öffentliches und anschließend internes auf dem jährlichen Bibliothekartag. In Düsseldorf haben wir uns mit den Themen elektronisches Pflichtexemplarrecht sowie den elektronischen Regionaldokumentationen BOA (Baden-Württembergisches Online Archiv) und eldoweb (Rheinland-pfälzischer Archivserver) in öffentlicher Sitzung beschäftigt.

Chance und Herausforderung der AG der Regionalbibliotheken ist die Heterogenität der Mitgliedsbibliotheken. Aus den unterschiedlichsten Interessenlagen und regionalen Problemstellungen heraus gilt es, die verbindenden Themen herauszukristallisieren. Hierzu hat sich die AG ein Arbeitsprogramm gegeben, das derzeit umgesetzt wird. Hier lassen sich folgende Schwerpunkte benennen:

\section{Elektronisches Pflichtexemplar}

Es herrscht innerhalb der AG Einigkeit darüber, dass der Nachweis, die ErschlieBung, die langfristige Archivierung und eine angemessene rechtliche Regelung des Pflichtexemplarrechts für Netz- oder Onlinepublikationen in unkörperlicher Form von deutlich zunehmender Bedeutung für unsere Bibliotheken sind. Die AG hat daher 2002 eine Arbeitsgruppe etabliert, die sich mit der Ausgestaltung und der Abstimmung mit der Deutschen Bibliothek bezüglich des elektronischen Pflichtexemplarrechts für nichtkörperliche Medien beschäftigt hat. Ein Musterentwurf wurde Anfang 2004 vorgelegt und im November 2004 von der KMK-AG Bibliotheken befürwortet. Eine Unter-AG hat darüber hinaus Sammelrichtlinien mit einer Vielzahl von Beispielen erarbeitet, die im Bibliotheksdienst veröffentlicht wurden. Beispiele für die Sammlung und Erschließung solcher Materialien 
sind edoweb (LB Koblenz) und BOA (WLB und BLB). Da zur Zeit die Novellierung auf Bundesebene in Arbeit ist und auf Länderebene geeignete gesetzliche Regelungen noch erfolgen müssen, bleibt die Pflichtexemplarregelung in den nächsten Jahren ein wichtiges Thema, ebenso die regionale Sammeltätigkeit. In diesem Zusammenhang sind für die AG ebenfalls die Fragen der Langzeitarchivierung und des Urheberrechts (hier insbesondere die öffentliche Zugänglichmachung und die Vervielfältigung) von Interesse.

\section{Historisches Erbe}

Die Mitgliedsbibliotheken bewahren - wie allgemein bekannt - außerordentliche Schätze. Es ist daher selbstverständlich, dass die AG in der Vergangenheit Themen aufgegriffen hat und auch künftig diskutieren wird, die hiermit in Zusammenhang stehen. Hierzu gehören u.a. Öffentlichkeitsarbeit, Kulturmanagement, Bestandserhaltung, Forschungsbibliothek, Digitalisierung etc.

\section{Zielgruppenarbeit}

Regionalbibliotheken haben zwar einen klaren regionalen Bezug, doch die Nutzerschaft ist zumeist heterogen und nicht klar abzugrenzen. Thema war und wird sein, wie die Regionalbibliotheken ihre Nutzer erreichen. Beispielsweise wurde über die Angebote zur Vermittlung von Informationskompetenz an Schüler und andere Nutzergruppen referiert.

\section{BIX-Regionalbibliotheken}

Die AG hat im Frühjahr 2004 eine Arbeitsgruppe ins Leben gerufen, die sich mit der Frage und der Ausgestaltung eines BIX für Regionalbibliotheken beschäftigt. Im Sommer 2004 wurde ein Kategorienschema mit Definitionen vorgelegt, das auf der Herbstsitzung in Trier den Mitgliedern zur Diskussion vorgestellt wurde. Auf dem Düsseldorfer Bibliothekartag 2005 wurde nach erneuter intensiver nicht-öffentlicher Diskussion beschlossen, an diesem Entwurf eines BIX-Regionalbibliotheken weiter zu arbeiten. Auf der Kasseler Herbstsitzung im Oktober 2005 wurde ein umfangreicher Pretest mit den Daten des Jahres 2004 ausgewertet. Hauptproblem eines BIX-Regionalbibliotheken ist die Heterogenität der Mitgliedsbibliotheken, die nur schwer in ein Kategorienschema zu bringen und dann vergleichbar gemacht werden können. Dennoch scheint es möglich zu sein, wesentliche Leistungsindikatoren zu definieren. Der nächste Schritt ist daher, die erarbeiteten Indikatoren so präzise zu definieren, dass sie von allen künftigen Teilnehmern einheitlich angewendet werden. Die AG der Regionalbibliotheken hat sich darauf verständigt, dass mit den Auswertungen insbesondere vergleichbare 
Regionalbibliotheken hinsichtlich der Einführung von Best-Practice-Lösungen sich gegenseitig unterstützen.

\section{Kooperations- und Integrationsmodelle}

Viele Regionalbibliotheken haben in den vergangenen Jahrzehnten ihre Selbständigkeit verloren, andere arbeiten eng mit den lokal vorhandenen Fachhochschulen oder Universitäten zusammen. Es liegen unterschiedlichste Integrations- und Kooperationsmodelle vor. Ebenso vielfältig sind die Erfahrungen. Da von Unterhaltsträgern unter Kostengesichtspunkten vermehrt und intensiv solche Modelle nicht nur ins Gespräch gebracht sondern konkret erzwungen werden, gilt es, hierüber einen Erfahrungsaustausch zu erhalten. Dies wird ein Schwerpunkt der Kasseler Herbstsitzung der AG der Regionalbibliotheken sein.

\section{Zusammenfassung}

Mit der AG haben sich die Regionalbibliotheken ein Forum für den Austausch über gemeinsame Interessen und für die Außendarstellung geschaffen. In Zeiten, in denen sich die Politik gerne verbal aber weniger finanziell für regionalbibliothekarische Funktionen engagiert, ist dieser Austausch um so wichtiger. Der hohe Organisationsgrad der Arbeitsgemeinschaft der Regionalbibliotheken scheint dies zu bestätigen.

Weitere Informationen über die AG sind über die Homepage des DBV unter www.regionalbibliotheken.de/regiobibo zu finden. 